\title{
Management of radiation-induced valvular heart disease due to Hodgkin's Lymphoma in the modern era
}

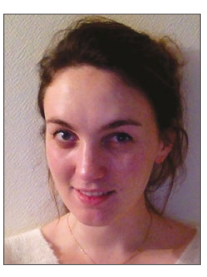

Elise Paven ${ }^{1}, \mathrm{MD}$; Claire Cimadevilla ${ }^{2}, \mathrm{MD}$; Marina Urena ${ }^{1}, \mathrm{MD}, \mathrm{PhD}$; Marie-Pierre Dilly ${ }^{3}, \mathrm{MD}$; Patrick Nataf $^{2}, \mathrm{MD}$; Richard Raffoul ${ }^{2}, \mathrm{MD}$; Dominique Himbert ${ }^{1}, \mathrm{MD}$; Alec Vahanian ${ }^{1,4,5}$, MD; David Messika-Zeitoun ${ }^{1,4,5 *}, \mathrm{MD}, \mathrm{PhD}$

1. Department of Cardiology, Assistance Publique - Hôpitaux de Paris, Bichat Hospital, Paris, France; 2. Department of Cardiac Surgery, Assistance Publique - Hôpitaux de Paris, Bichat Hospital, Paris, France; 3. Department of Anaesthesiology, Assistance Publique - Hôpitaux de Paris, Bichat Hospital, Paris, France; 4. INSERM U1148, Bichat Hospital, Paris, France, 5. University

Paris VII, Paris, France

\section{Introduction}

Irradiation doses used in the early days to cure Hodgkin's Lymphoma are responsible for late cardiac complications including valvular heart disease (VHD). Radiation-induced VHD (RI-VHD) is a major challenge for cardiac surgeons as they may face hostile thorax and myocardial damage which are often underestimated $^{1}$. The last decade has seen the remarkable development of transcatheter aortic valve implantation (TAVI) and, more recently, transcatheter mitral valve implantation (TMVI) ${ }^{2}$. In the present study, we reviewed all consecutive patients with symptomatic RI-VHD due to Hodgkin's Lymphoma who underwent either a surgery, a TAVI or a TMVI at our institution.

Editorial, see page 1735

\section{Methods}

Consecutive patients diagnosed with severe mitral and/or aortic RI-VHD due to Hodgkin's Lymphoma who underwent a surgical or transcatheter intervention at Bichat Hospital were retrospectively enrolled. The decision to perform a transcatheter or a surgical intervention was left to the Heart Team. Follow-up was obtained through visits to clinics or phone calls to the patients/referring cardiologists and was complete up to July 2016. Comparisons between the groups were performed using the Student's t-test, Mann-Whitney test, chi ${ }^{2}$ test or Fisher's exact test, as appropriate. Survival rates were assessed using the Kaplan-Meier method, and comparison according to therapy by means of log-rank test.

\section{Results}

Between January 2006 and January 2016, 37 patients were admitted and treated in Bichat Hospital for RI-VHD due to Hodgkin's disease. Median interval between radiotherapy and transcatheter/ surgical therapy was 32 years (27-41). Twenty-one patients $(57 \%)$ presented with an isolated aortic valve disease, five (13\%) with an isolated mitral disease and $11(30 \%)$ with a combined aortic and mitral valve disease.

Sixteen patients $(43 \%)$ were referred to surgery, 17 patients (46\%) underwent a TAVI and four patients (11\%) a TMVI. All interventions were successful. No combined valvular intervention was performed in the transcatheter groups $(0 \%)$ compared to $38 \%$ in the surgical group ( $\mathrm{p}<0.001)$. Among the 16 patients who were operated on, nine patients $(56 \%)$ had an isolated aortic valve disease, one patient $(6 \%)$ an isolated mitral valve disease and six 
patients (38\%) both aortic and mitral valve diseases. A coronary artery bypass graft was also performed in seven patients (44\%), a tricuspid annuloplasty in one patient and an interatrial patch in one patient. A moderate to severe associated mitral valve disease was observed in five patients $(29 \%)$ who underwent a TAVI but this was disregarded.

Five patients $(14 \%)$ died in the postoperative period. The difference did not reach statistical significance due to the limited sample size. In-hospital complications were also not different between groups (Table 1). In-hospital length of stay tended to be shorter in the transcatheter groups $(p=0.07)$ but achieved statistical significance $(\mathrm{p}=0.05)$ when surgical and TAVI groups were compared. No patients were lost to follow-up. Ten patients (31\%) died after hospital discharge. One-year mortality was $24 \%$ in the overall population, $25 \%$ in the surgical group, $18 \%$ in the TAVI group and $50 \%$ in the TMVI group $(\mathrm{p}=0.18)$ (Figure 1). Symptoms improved in most patients and $91 \%$ of survivors were in NYHA Class I/II.

\section{Discussion}

Outcome was poor with a survival rate $<50 \%$ at five years despite successful valvular interventions. We only enrolled patients with mediastinal irradiation for Hodgkin's Lymphoma and excluded other causes of mediastinal radiation such as for breast cancers. Extensive mediastinal irradiation, as performed for Hodgkin's disease, is associated with the worst outcomes ${ }^{3}$.

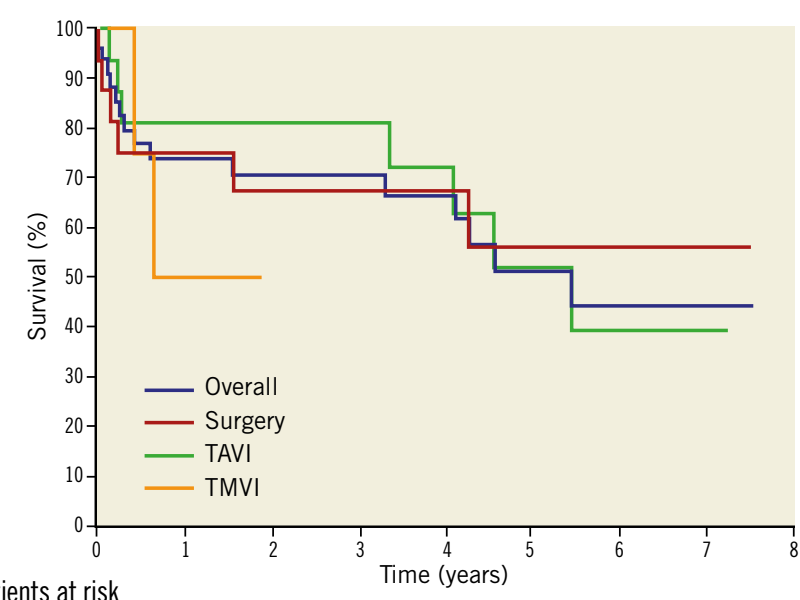

Patients at risk

$\begin{array}{lrrrrrrrr}\text { Overall } & 37 & 24 & 22 & 15 & 14 & 8 & 7 & 3 \\ \text { Surgery } & 16 & 11 & 10 & 6 & 6 & 5 & 5 & 2 \\ \text { TAVI } & 17 & 11 & 10 & 9 & 6 & 3 & 2 & 1\end{array}$

TMVI

Figure 1. Survival in the overall population and according to the type of intervention (surgery, transcatheter aortic valve implantation [TAVI] and transcatheter mitral valve implantation [TMVI]).

Less than half of our population was operated on, illustrating the shift in indications and Heart Team decisions. Patients with isolated aortic valve disease underwent a TAVI preferentially. It is worth noting that, in the TAVI group, several patients also presented with associated mitral valve disease that was disregarded.

Table 1. Characteristics of the population and in-hospital complications overall and according to therapy.

\begin{tabular}{|c|c|c|c|c|c|}
\hline & Overall $(n=37)$ & Surgery $(n=16)$ & TAVI $(n=17)$ & TMVI $(n=4)$ & $p$-value (3 groups) \\
\hline Age (years) & 56 [50-65] & $56[50-66]$ & 63 [53-67] & 51 [44-59] & 0.16 \\
\hline Male gender & $26(70 \%)$ & $9(56 \%)$ & $14(82 \%)$ & $3(75 \%)$ & 0.25 \\
\hline History of coronary artery disease & $20(54 \%)$ & $4(25 \%)$ & $12(71 \%)$ & $4(100 \%)$ & 0.0005 \\
\hline Previous cardiac surgery & $9(24 \%)$ & $1(6 \%)$ & $5(30 \%)$ & $3(75 \%)$ & 0.01 \\
\hline Atrial fibrillation & $10(27 \%)$ & $3(19 \%)$ & $6(35 \%)$ & $1(25 \%)$ & 0.56 \\
\hline Respiratory disease & $20(54 \%)$ & 7 (44\%) & $9(53 \%)$ & $4(100 \%)$ & 0.13 \\
\hline NYHA Class III/IV & $28(76 \%)$ & $12(75 \%)$ & $12(71 \%)$ & $4(100 \%)$ & 0.47 \\
\hline Logistic EuroSCORE 1 (\%) & $4[3-11]$ & $4[2-6]$ & $4[2-11]$ & $16[5-34]$ & 0.0005 \\
\hline EuroSCORE $2(\%)$ & $3[2-5]$ & $3[2-4]$ & $3[1-5]$ & 10 [3-15] & 0.004 \\
\hline Charlson comorbidity index & $5[4-6]$ & $4.5[3-6]$ & $5[4-6]$ & $8[6-11]$ & 0.008 \\
\hline Left ventricular ejection fraction (\%) & 57 [42-63] & $60[35-63]$ & 57 [49-63] & 48 [40-53] & 0.37 \\
\hline Systolic pulmonary artery pressure $(\mathrm{mmHg})$ & 41 [39-55] & $50[38-51]$ & 41 [34-48] & $60[45-67]$ & 0.15 \\
\hline Creatinine clearance $\left(\mathrm{mL} / \mathrm{min} / 1.73 \mathrm{~m}^{2}\right)$ & 84 [64-95] & $84[72-106]$ & $81[65-91]$ & 74 [32-98] & 0.60 \\
\hline In-hospital death & $5(14 \%)$ & $4(25 \%)$ & $1(6 \%)$ & $0(0 \%)$ & 0.19 \\
\hline Median in-hospital stay (days) & 9 [7-17] & 14 [10-17] & 7 [6-15] & $6[5-12]$ & 0.07 \\
\hline Postoperative stroke & $1(3 \%)$ & $0(0 \%)$ & $1(6 \%)$ & $0(0 \%)$ & 0.56 \\
\hline Tamponade & $2(5 \%)$ & $2(13 \%)$ & $0(0 \%)$ & $0(0 \%)$ & 0.23 \\
\hline Postoperative congestive heart failure & $11(30 \%)$ & $7(47 \%)$ & $2(12 \%)$ & $2(50 \%)$ & 0.07 \\
\hline Postoperative vascular complications & $5(14 \%)$ & - & $4(23 \%)$ & $1(25 \%)$ & 0.55 \\
\hline Postoperative kidney injury & $4(11 \%)$ & $1(7 \%)$ & $2(12 \%)$ & $1(25 \%)$ & 0.52 \\
\hline Major bleeding & $5(14 \%)$ & $3(20 \%)$ & $1(6 \%)$ & $1(25 \%)$ & 0.38 \\
\hline Postoperative shock & $9(24 \%)$ & $5(33 \%)$ & $2(12 \%)$ & $2(50 \%)$ & 0.18 \\
\hline
\end{tabular}

Categorical data are presented as the number of events and percent in each group. Quantitative data are presented as median and interquartile range. 
Transcatheter procedures appeared a reasonable alternative to surgery. In-hospital mortality tended to be lower and length of stay shorter. TMVI was performed in patients presenting with severe degenerative mitral stenosis due to massive annular calcifications. These patients presented the worst preoperative state. Nevertheless, the procedural and immediate outcomes appeared encouraging. Half of the patients died within one year, but mortality was related to the severe preoperative condition of the patients and not to the procedure. Thus, TMVI appears to be an interesting alternative to surgery in these very challenging surgical candidates.

\section{Limitations}

Several limitations need to be underlined. This was a singlecentre retrospective registry and our sample size was relatively small, especially the TMVI subset. However, our series was homogenous and, in contrast to most studies, we enrolled patients exclusively with RI-VHD due to Hodgkin's disease. Only patients with severe valvular heart disease who underwent a transcatheter or surgical procedure were enrolled and the three groups were not comparable.

\section{Conclusions}

In this homogeneous and contemporary series of patients with RI-VHD due to Hodgkin's disease, we observed that overall prognosis remained poor, with a $50 \%$ five-year survival rate despite successful management of the valvular disease. Transcatheter interventions appeared a reasonable alternative to surgery and may possibly be the preferred option in case of isolated valve disease, although confirmation is needed for mitral valve interventions.

\section{Impact on daily practice}

The present study suggests that, in patients with isolated radiation-induced valvular heart disease due to Hodgkin's Lymphoma, transcatheter intervention may be the preferred option, although confirmation is needed at the mitral valve level.

\section{Conflict of interest statement}

D. Himbert is consultant and proctor for Edwards Lifesciences and Medtronic. A. Vahanian has received consulting honoraria from Edwards Lifesciences, Abbott, and Valtech. D. Messika-Zeitoun has received consulting honoraria/research grants from Edwards Lifesciences, Abbott, Cardiawave, Mardil and Valtech. The other authors have no conflicts of interest to declare.

\section{References}

1. Handa N, McGregor CG, Danielson GK, Daly RC, Dearani JA, Mullany CJ, Orszulak TA, Schaff HV, Zehr KJ, Anderson BJ, Schomberg PJ, Puga FJ. Valvular heart operation in patients with previous mediastinal radiation therapy. Ann Thorac Surg. 2001;71:1880-4.

2. Himbert D, Bouleti C, Iung B, Nejjari $M$, Brochet $E$, Depoix JP, Ghodbane W, Fassa AA, Nataf P, Vahanian A. Transcatheter valve replacement in patients with severe mitral valve disease and annular calcification. J Am Coll Cardiol. 2014; 64:2557-8

3. Chang AS, Smedira NG, Chang CL, Benavides MM, Myhre U, Feng J, Blackstone EH, Lytle BW. Cardiac surgery after mediastinal radiation: extent of exposure influences outcome. J Thorac Cardiovasc Surg. 2007;133:404-13. 